\title{
Research Article \\ Performance Evaluation of MBA-OLSR Routing Protocol for MANETs
}

\author{
Waheb A. Jabbar, Mahamod Ismail, and Rosdiadee Nordin \\ Department of Electrical, Electronic and System Engineering, Universiti Kebangsaan Malaysia, 43600 Bangi, Selangor, Malaysia
}

Correspondence should be addressed to Waheb A. Jabbar; waheb@ieee.org

Received 8 April 2014; Accepted 27 June 2014; Published 15 July 2014

Academic Editor: Lixin Gao

Copyright (C) 2014 Waheb A. Jabbar et al. This is an open access article distributed under the Creative Commons Attribution License, which permits unrestricted use, distribution, and reproduction in any medium, provided the original work is properly cited.

\begin{abstract}
This paper proposes a multipath battery aware routing protocol, namely, MBA-OLSR, based on OLSRv2 and its multipath extension. The proposed approach considers the remaining battery energy of the nodes for calculating the initial cost of the multiple links between source-destination pairs. The MBA-OLSR aims to construct energy efficient routes and extend the network lifetime to avoid network failure due to battery exhaustion. The protocol performance is evaluated in different simulation scenarios of wireless mobile ad hoc networks (MANETs) environment. The simulation results demonstrate that our approach can achieve longer network lifetime and lower energy cost per packet. In addition, the results show improvement in terms of packet delivery ratio and end-toend delay.
\end{abstract}

\section{Introduction}

Mobile ad hoc networks (MANETs) depict complex distributed systems that comprise wireless mobile nodes. These nodes can freely and dynamically self-organize into arbitrary and temporary ad hoc network topologies, thus allowing people and devices to seamlessly internetwork in areas with no preexisting communication infrastructure, for example, disaster recovery environments [1]. The routing protocols are used to route the packets from the source node to the destination. These routing protocols should be efficient in terms of quality of service (QoS) metrics and energy consumption to guarantee the data transmission over the wireless medium [2]. The internet engineering task force (IETF) has developed four routing protocols mainly for MANET [3]: ad hoc ondemand vector: (AODV), dynamic source routing (DSR), topology dissemination based on reverse-path forwarding (TBRPF), and optimized link state routing (OLSR) [4]. The efforts of the IETF are being made to standardize the OLSRv2 $[5,6]$, a successor to OLSR, and dynamic MANET ondemand (DYMO) which is currently known as AODVv2, a successor to both AODV and DSR routing protocols [3].

The limited capacity of mobile nodes batteries represents a constraint for MANETs. Therefore, the design of energy efficient routes is a substantial need for saving energy and extending batteries and network lifetime. The failure of nodes caused by the battery exhaustion restricts the node's ability to transmit and receive or relay packets to others as an intermediate node. Thus, routing a packet between sourcedestination pairs requires an adequate number of alive nodes with enough energy to forward the packets between them.

The multipath routing protocols are proposed to address some routing issues, for example, scalability, and transmissions instability. multi-path-optimized link state routing protocol (MP-OLSR) is proposed in [7] as a multipath extension to OLSRv2 [5]. In the MP-OLSR protocol, the multipath Dijkstra algorithm is proposed to obtain multiple paths from a source to a destination. In addition, route recovery and loop detection are implemented in the MP-OLSR in order to improve QoS regarding OLSR. The algorithm exploited two cost functions for the link cost between nodes to generate node-disjoint or link-disjoint paths. However, the MP-OLSR uses the number of hops as a metric to find the best path to the destination and the costs of all links between sourcedestination pair links are initialized to one (1) as shown in Figure 1. The costs of the links between two nodes are treated equally; that is, the path with the least hop counts (shortest path) is considered as the best path. However, this 


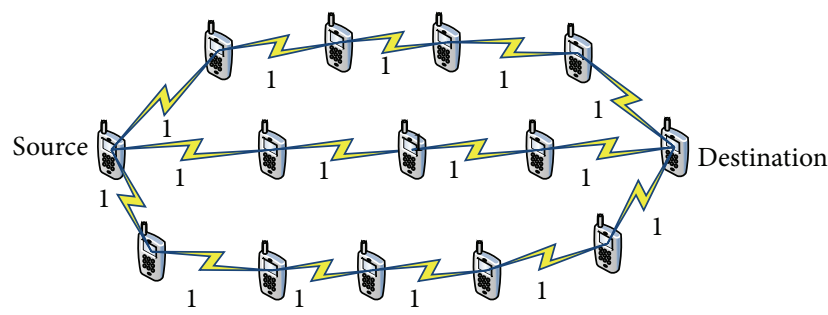

FIGURE 1: MP-OLSR multiple paths in initial state cost function.

measurement is not always correct because its choice can lead to a significant reduction of network performance when the network operates for a long time or if there are some nodes in the network with low battery levels. In such situations, some batteries of the nodes will be drained when these nodes are always chosen in the shortest path to the destination. In other words, the shortest path is not always being the efficient path. Thus, the evaluation of links energy efficiency is a key factor to be considered.

In this paper, we propose an approach for route computation in the MP-OLSR [7]. The enhanced protocol is known as a multipath battery aware OLSR (MBA-OLSR) [8]. The proposed approach considers the residual battery energy of the nodes during the route computation as a parameter in the link cost function. Then the multipath Dijkstra algorithm can use the cost based on the residual battery energy as the initial cost of the links between each pair of nodes to find the best path to a destination instead of number of hops which is used in the original MP-OLSR.

In the next section, we present an overview of both versions of the OLSR and MP-OLSR routing protocols. In Section 3, the related works are highlighted and followed by the proposed MBA-OLSR protocol in Section 4. The simulation models with the performance metrics are discussed in Section 5. Section 6 presents the simulation results and discussion with comparison. Finally, a conclusion is drawn up in Section 7.

\section{Background}

An overview of both versions of OLSR is presented in this section. Then the concept and functionalities of MP-OLSR are discussed.

2.1. OLSR and OLSR Version 2. Currently, OLSR [4] is the most employed and leading proactive routing protocol in MANETs. The first version of OLSR (OLSRv1) has been standardized as an experimental RFC 3626 [4]. It works in a proactive manner; that is, topology information is exchanged between the nodes on a periodic basis. The core optimization of OLSR is to minimize the control traffic by selecting a small number of nodes, known as multipoint relays (MPR) which is an improved flooding mechanism for topological information.

The second version of OLSR is OLSRv2 [5]. It is an update and successor to OLSRv1. It holds the same basic mechanisms and algorithms of OLSRv1; however, OLSRv2

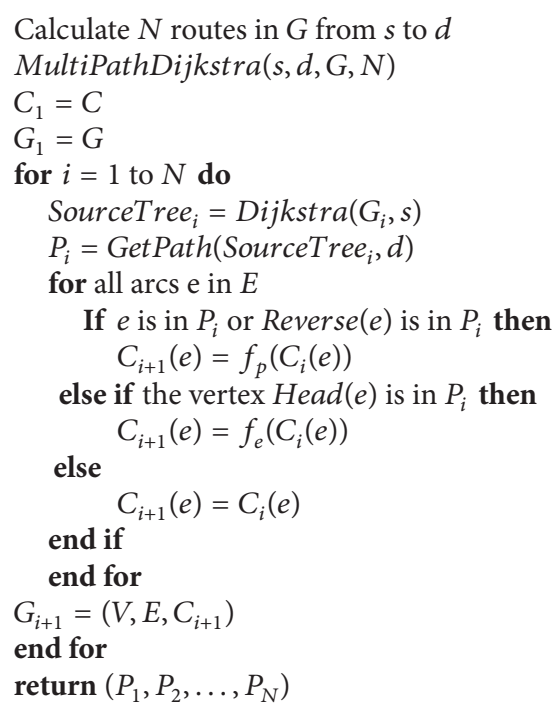

Algorithm 1: The multipath Dijkstra algorithm.

provides an even more flexible signaling framework and some simplification of the exchanged messages between nodes. It also accommodates both IPv4 and IPv6 addresses in a compact fashion. The OLSRv2 is developed for MANETs. Basically, it modifies the OLSRvl by using and extending the following generalized building blocks:

(i) the MANET neighborhood discovery protocol (NHDP) defined in [RFC6130] [9],

(ii) the generalized MANET packet/message format [RFC5444] [10],

(iii) the TLVs as specified in [RFC5497] [11],

(iv) optionally, message jitter as specified in [RFC5148] [12].

Now the OLSRv2 is in its final stage of standardization [13].

2.2. MP-OLSR. In this subsection, we discuss the concept and functionalities of the MP-OLSR [7]. It is a hybrid multipath routing protocol which is based on OLSRv2. It is proposed to improve QoS, load-balancing, and energy conservation. It takes an advantage of the MPR mechanism to flood the network with control traffic information and includes a major modification of the Dijkstra algorithm as detailed in Algorithm 1. The MP-OLSR changes the OLSR proactive behavior into on-demand route computation and becomes a source routing protocol with two cost functions to produce multiple disjoint or nondisjoint paths. The incremental functions cost function $F_{p}$ and cost function $F_{e}$ are used at each step to get a disjoint path between $s$ and $d$. The $F_{p}$ cost function is used to increase the costs of the arcs that belong to the previous path $P_{i}$ (or the opposite arcs belonging to it). This will make future paths tend to use different arcs. The $F_{e}$ cost function is used to increase the costs of the arcs that lead to the vertices of the previous path $P_{i}$. 
To maintain several paths for the same source-destination pair, there are two main phases: (i) topology sensing and (ii) route computation. The topology sensing phase includes link sensing, neighbor detection, and topology discovery. The nodes obtain a partial topology map just like that in the OLSR based on the periodic exchange of HELLO and TC messages. However, MP-OLSR nodes do not construct routing tables. Throughout the route computation phase, these nodes calculate multiple paths (one is a primary path and the others are secondary paths) and reach any other node in the network following an on-demand scheme and based on topology sensing information. Furthermore, the MP-OLSR has two auxiliary phases: route recovery and loop detection to improve its performance. Also, it is compatible with standardized OLSR by using the IP source routing. The queue length as a link metric for computation of routing paths is also proposed in MP-OLSR (MPQ-OLSR) to reflect the link quality faithfully [14]. The average queue length has an essential effect on the delay.

\section{Related Works}

Several studies on energy efficient routing consider the available energy of nodes to take the routing decisions. EEOLSR [15] is an enhanced version of OLSR for extending the network lifetime without loss of performance. The authors modified the multipoint relay (MPRs) selection mechanism of the OLSR protocol based on the EA-willingness concept mechanisms by considering the energy state of the node during MPR selection. However, the EEOLSR has not considered the residual battery for route computation. The authors in [16] proposed OLSRE, an enhanced version energy efficient routing based on OLSR. In OLSRE, the energy consumption is taken into account during packet routing by calculating the cost of packet transmission. However, this protocol has a high overhead and it does not consider a node's residual energy.

Another approach of energy efficient OLSR-based routing protocol is the OLSR_EA [17] with the autoregressive integrated moving average time series method. The purpose of this is to measure and predict perinterval energy consumption. The authors developed a composite energy cost by considering residual energy and the consumed transmission power of each node and used this compound energy index as the energy routing metric. However, in the OLSR_EA, there is a higher chance of collision if a relay node has a longer transmission range than surrounded nodes and, therefore, it is not preferred in large networks.

In [18], the authors proposed the OLSRM protocol based on the standardized OLSR. They have tried to make it energy efficient by proposing an efficient neighbor selection based on node's residual energy and drain rate. They have considered the multipath with the source routing concept for route selection. Several works in the literature have been proposed to optimize the energy consumption of OLSR; however, all of them are based on the standardized first version of OLSR (OLSRv1). To the best of our knowledge, none of the researches in the literature consider the energy efficiency of OLSRv2 [8], the successor to OLSRv1, or its multipath extension MP-OLSR [9].

\section{The Proposed MBA-OLSR}

The MP-OLSR applies the number of hops as the link cost metric for transmitting packets. Initially, it sets the cost for all links between the source and destination to "1" (see Figure 1) which can either lead the congestion on a specific path or rise in the energy expenditure of particular intermediate nodes. For the proposed MBA-OLSR [8], the same multipath Dijkstra algorithm as shown in Algorithm 1 [7] was utilized. However, the initial link cost of each link was calculated based on the residual battery of both nodes for each link.

Since the topology information in the OLSR and MPOLSR is exploited by exchanging HELLO and TC messages, we need to modify these messages for MBA-OLSR and attach the residual energy information of the node to them. The type-length-value (TLV) mechanism of OLSRv2 [11] allows to add an additional TLV for the residual battery information. These modifications make other nodes in the network aware of the residual battery energy information in the local node. As shown in Figure 2, a TLV_Residual_Battery is defined. It is composed of 3-byte TLV header and 2-byte for residual battery information. The length is normalized into a double between $[0,65535]$. Then, the TLV can be carried by the HELLO and TC messages and broadcasted to the whole network. Upon the reception of HELLO or TC messages, the residual energy of the battery is extracted from the TLV_Residual_Battery. A cost function is used to estimate the link cost between two nodes based on their residual battery's energy as defined in the following:

$$
\begin{gathered}
\mathrm{RB}_{i j}=\frac{\mathrm{RB}_{i}}{\mathrm{RB}_{i}+\mathrm{MRB}}+\frac{\mathrm{RB}_{j}}{\mathrm{RB}_{j}+\mathrm{MRB}}, \\
\operatorname{LinkCost}_{i j}=1+k * \frac{1}{e^{\mathrm{RB}_{i j}}},
\end{gathered}
$$

where $M R B$ is the maximum battery capacity, $R_{i}$ and $\mathrm{RB}_{j}$ are the residual battery energy of the nodes $i$ and $j$, respectively, and $\mathrm{RB}_{i j}$ is the ratio of residual battery energy of both nodes $i$ and $j$. $K$ is the weight factor to optimize the impact of different remaining battery energy on the link cost. This parameter is selected to investigate the behavior of the protocol different weight factor values.

If multiple paths are available, initially, the cost of all links is equal to the link cost obtained from (2). The next hop nodes are selected based on their weights which are updated based on the link cost to them. The nodes with the highest level of residual battery energy will be connected by the links with minimum cost and construct the best route to the destination. The multipath computation process is explained in the flow chart in Figure 3.

\section{Simulations}

5.1. Simulation Tools. EXata 3.1 simulator [19] is employed to study and analyze the performance of MBA-OLSR. It is used to conduct extensive simulations to evaluate the energy consumption and QoS metrics of the MBA-OLSR and compare it with the original MP-OLSR routing protocol in different scenarios. The EXata communication simulation 


\begin{tabular}{|c|c|c|c|}
\hline$\leftrightarrow$ Header information & $-\langle$ msg_TLV_buffer $\rangle\langle 12$ bytes $\rangle$ & $\leftarrow$ information & $\longleftarrow$ Neighbor \\
\hline $\begin{array}{l}\langle\text { msg_header }\rangle \\
\langle\text { msg_header_info }\rangle\end{array}$ & 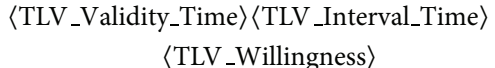 & $\begin{array}{l}\langle\text { Local_addr } \\
\text { _block_buff }\rangle\end{array}$ & $\begin{array}{c}\langle\text { Addr_block } \\
\text { _buff }\rangle\end{array}$ \\
\hline
\end{tabular}

(a) Original TLV added to the HELLO message

\begin{tabular}{|c|c|c|c|}
\hline$\leftarrow$ Header informati & $-\langle$ msg_TLV_buffer $\rangle\langle 17$ bytes $\rangle$ & information & $\succ \leftarrow \begin{array}{c}\text { Neighbor } \\
\text { intormation }\end{array}$ \\
\hline $\begin{array}{l}\langle\text { msg_header }\rangle \\
\langle\text { msg_header_info }\rangle\end{array}$ & 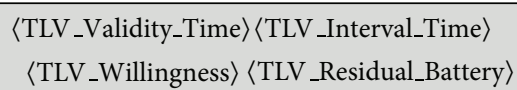 & $\begin{array}{l}\langle\text { Local_addr } \\
\text { _block_buff }\rangle\end{array}$ & $\begin{array}{c}\langle\text { Addr_block } \\
\text { _buff }\rangle\end{array}$ \\
\hline
\end{tabular}

(b) Modified TLV with TLV_Residual_Battery added to the HELLO message

\begin{tabular}{|c|c|c|c|}
\hline \multicolumn{2}{|c|}{$\leftarrow$ Header information $\rightarrow \longleftarrow\langle$ msg_TLV_buffer $\rangle\langle 12$ bytes $\rangle$} & \multicolumn{2}{|c|}{$\underset{\text { information }}{\text { Local }} \longrightarrow \stackrel{\text { Neighbor }}{\text { intormation }}$} \\
\hline $\begin{array}{l}\langle\text { msg_header }\rangle \\
\langle\text { msg_header_info }\rangle\end{array}$ & $\begin{array}{c}\langle\text { TLV_Validity_Time }\rangle\langle\text { TLV_Content_seq_number }\rangle \\
\langle\text { TLV_Interval_Time }\rangle\end{array}$ & $\begin{array}{l}\text { 〈Local_addr } \\
\text { _block_buff }\rangle\end{array}$ & $\begin{array}{c}\langle\text { Addr_block } \\
\text { buff }\rangle\end{array}$ \\
\hline
\end{tabular}

(c) Original TLV added to the TC message

\begin{tabular}{|c|c|c|c|}
\hline$\leftarrow$ Header information & $-\langle$ msg_TLV_buffer $\rangle\langle 17$ bytes $\rangle$ & $\overleftarrow{\text { information }}_{\text {Local }}$ & $\begin{array}{l}\text { Neighbor } \\
\text { information }\end{array}$ \\
\hline $\begin{array}{l}\langle\text { msg_header }\rangle \\
\langle\text { msg_header_info }\rangle\end{array}$ & $\begin{array}{c}\langle T L V \text { TValidity_Time }\rangle\langle T L V \text { _Content_seq_number }\rangle \\
\langle T L V \text { Interval_Time }\rangle\langle T L V \text { Residual_Battery }\rangle\end{array}$ & $\begin{array}{l}\langle\text { Local_addr } \\
\text { _block_buff }\rangle\end{array}$ & $\begin{array}{l}\langle\text { Addr_block } \\
\quad \text { buff }\rangle\end{array}$ \\
\hline
\end{tabular}

(d) Modified TLV with TLV_Residual_Battery added to the TC message

FIgURE 2: Type-length-value (TLV) added to HELLO and TC messages in MBA-OLSR.

platform is a network emulator that is integrated into the well-known QualNet network simulator, which is widely used in academic research and industry. The EXata simulator platform offers a high level of reliability and scalability simulations for wireless communication.

5.2. Network Model. Basically, the MANET network can be modeled using graph $G(V, E)$, where $V$ is the set of nodes representing the mobile devices and $E$ is a set of arcs; each arc models the intersection of two devices' communications range [20]. Each node $v \in V$ can directly communicate with a set of neighboring devices within its communication range. Otherwise, it has to use a routing protocol to transmit packets to remote devices that are not adjacent neighbors. One of the possible paths in the graph $G(V, E)$ can be used to transmit the packets from the node $v$ to the destination node. Due to the node's mobility, the network topology frequently changes. Consequently, the cardinality of nodes in set $V$ remains the same throughout the period, whereas the cardinality of edges in $E$ keeps changing. The average hop length of $E$ increases with the number of nodes in set $V$ and this affects the performance of the routing protocol. Therefore, the network size is one of the major parameters in simulation studies of routing protocol evaluation in MANET. In our simulation, the number of nodes was set to 49 nodes as it is described in Section 5.8.

5.3. Energy Model. The battery energy of the mobile node is limited and node can only transmit a restricted number of bits. The maximum number of bits that can be transmitted is defined by the total battery energy divided by the required energy per bit. There are four states for the mobile node in a wireless network: transmit, receive, idle, and sleep. Each state consumes a particular amount of power $\left(P_{\text {transmit }}, P_{\text {receiv }}, P_{\text {idle }}\right.$, and $\left.P_{\text {sleep }}\right)$. In our simulation model, we used a generic radio energy model which is derived to estimate the consumed energy based on the circuitry power consumption and time spent in each state as follows:

$$
\begin{aligned}
E_{\text {trans }} & =P_{\text {transmit }} * t_{\text {trans }} \\
E_{\text {rec }} & =P_{\text {receiv }} * t_{\text {rec }} \\
E_{\text {idle }} & =P_{\text {idle }} * t_{\text {idle }} \\
E_{\text {sleep }} & =P_{\text {sleep }} * t_{\text {sleep }},
\end{aligned}
$$

where $t_{\text {trans }}, t_{\text {rec }}$, and $t_{\text {idle }}$ are the duration of time for each state. Therefore, the total energy consumption for a node to transmit and receive a packet can be calculated as shown in

$$
E_{\text {total }}=E_{\text {trans }}+E_{\text {rec }}+E_{\text {idle }} \text {. }
$$

We set the energy parameters in our simulation scenarios as listed in Table 1 based on [15].

5.4. Network Lifetime Model. The main purpose of energy efficient routing is to maximize the network lifetime. In this paper, we used the definition of the network lifetime [21] as duration of time until the first node in a network failed because the battery depleted. If all the nodes have the same initial energy levels, the node which dies first will be the one which expends the battery energy at the highest rate. If we want to extend the lifetime of the network, it is critical to include the residual battery energy into links cost updates. 


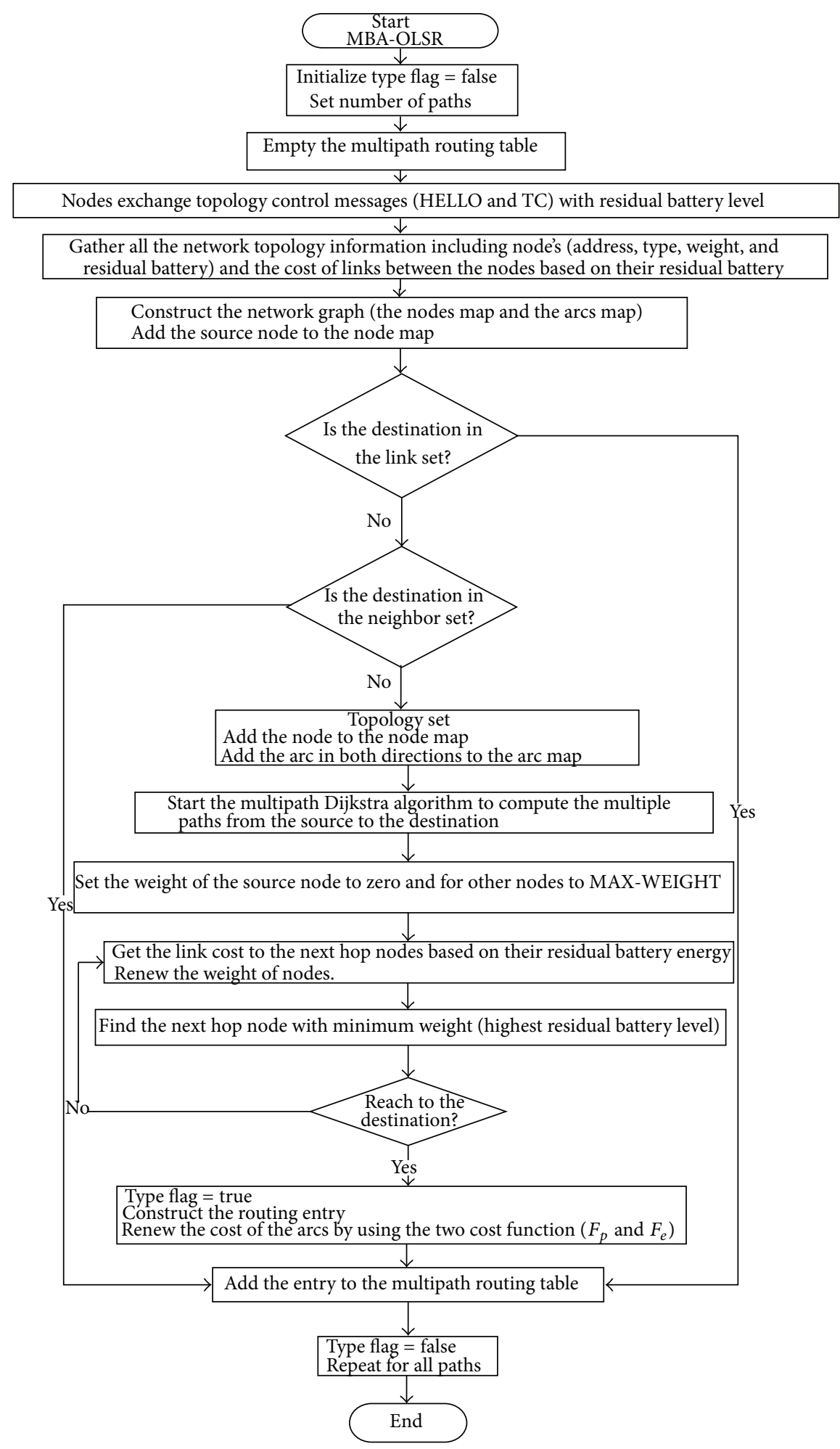

FIgURE 3: Flowchart for route computation in MBA-OLSR.

5.5. Linear Battery Model. Battery provides voltage and current for the components attached to the battery such as radio interfaces, CPU, memory blocks, and sensing core. A DC-DC converter regulates voltage for different components (see Figure 4). The battery is a repository of electrical charges which losses its charge when a load (electrical current) is taken off from it. The loss rate is a function of the load. The total energy consumed by the system per cycle is 
TABLE 1: Simulation parameters.

\begin{tabular}{lc}
\hline Parameter & Values \\
\hline Simulator & EXata 3.1 \\
Routing protocol & MBA-OLSR, MP-OLSR and \\
MPQ-OLSR & $1000 \mathrm{~m} \times 1000 \mathrm{~m}$ \\
Simulation area & 400 seconds \\
Simulation time & 49 \\
Number of nodes & $16 \mathrm{CBR}$ \\
Number of sources traffic & RWP $($ max speed $0-10 \mathrm{~m} / \mathrm{s}$, \\
Mobility model & pause time $50-350 \mathrm{sec})$ \\
Transport protocol & IPv 4 \\
Network protocol & $50000 \mathrm{bytes}$ \\
Priority input queue size & IEEE 802.11 \\
MAC protocol & PHY $802.11 \mathrm{~b}$ \\
Physical layer model & Generic $\left(P_{\text {transmit }}=1400 \mathrm{~mW}, P_{\text {receive }}=\right.$ \\
Energy model & 1000 mW, $P_{\text {Idle }}=0 \mathrm{~mW}$, and $P_{\text {Sleep }}=$ \\
Battery model & $0 \mathrm{~mW})$ \\
Data rate & Linear battery model \\
\hline
\end{tabular}

the sum of energies consumed by the radio transceivers $\left(E_{\text {Trans }}\right)$ protocol processor $\left(E_{\mathrm{CPU}}\right)$, the DC-DC converter $\left(E_{\mathrm{DC}}\right)$, and the efficiency losses in the battery $\left(E_{\mathrm{Bat}}\right)[19]$ :

$$
E_{\text {Cyclel }}=E_{\text {Trans }}+E_{\mathrm{CPU}}+E_{\mathrm{DC}}+E_{\text {Bat }} .
$$

The total energy consumed during the execution of the software on a given hardware architecture is the sum of the energies consumed during each cycle. The utilized model in our simulation is a simple linear model. It is based on the coulomb counting technique [22]. The coulomb counting technique accumulates the dissipated coulombs from the beginning of the discharge cycle and estimates the remaining capacity based on the difference between the accumulated value and a prerecorded full-charge capacity. This method can lose some of its accuracy under variable load conditions because it ignores the nonlinear discharge effect during the coulomb counting process. The battery charge monitoring interval is set to $1 \mathrm{sec}$ and the value of full battery capacity is set to 18 [mA.h] for the implemented linear battery model.

5.6. Mobility Model. Node mobility in a mobile multihop wireless network can be described by the node's speed as well as its pause duration. The average speed of the nodes in the network is an indicator of changing the network topology and the rate of links break as well as routing overhead. The random waypoint model (RWP) [23] is one of the most widely nd the value of full battery capacity is set used mobility models in MANET simulation. This model selects random destinations and speeds from 0 to $V_{\text {max }}$ for each node. After the nodes reach their selected destinations, they pause for a defined duration of time and then the process is repeated. In our simulation, we focused on the node's mobility to evaluate the performance of the proposed approach. The nodes pause

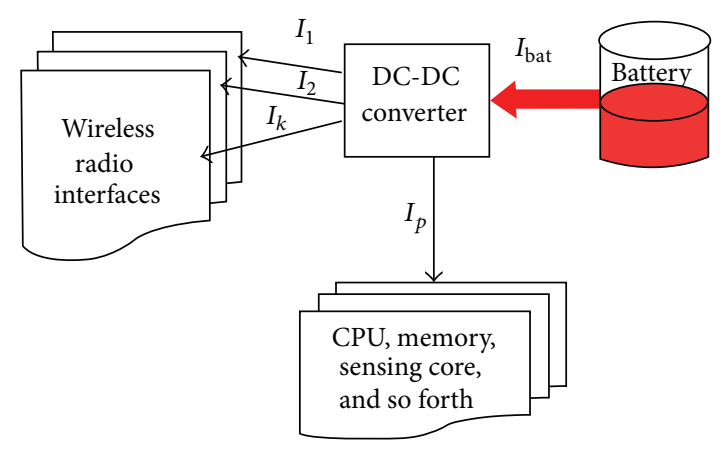

FIGURE 4: System level block of smart batteries [19].

time duration and maximum speed were considered in the two simulation scenarios. In the first scenario, the pause time duration of nodes was changed from $50 \mathrm{sec}$ to $350 \mathrm{sec}$. However, in the second scenario, the maximum speed $\left(V_{\max }\right)$ of nodes was changed from $1 \mathrm{~m} / \mathrm{s}$ to $10 \mathrm{~m} / \mathrm{s}$ as discussed in Section 6.

5.7. The Traffic Generation Model. The traffic model has a serious effect on the performance of the routing protocols and its execution [24]. Furthermore, the change in the traffic model parameters such as flow number, packet number, or packet size will impact the congestion level in the network. In this paper, we used the well-known UDP traffic generated by the constant bit rate (CBR) which generates traffic at a constant rate by transmitting packets of a fixed size at a fixed rate. It is commonly used to provide background traffic that impacts the performance of other applications being analyzed or to simulate generic multimedia traffic. The CBR can be used to simulate applications for which the end-systems require predictable response time and a static amount of bandwidth continuously available for the lifetime of the connection. These applications include services such as video-conferencing and telephony (voice services).

5.8. Simulation Environment and Parameters. In this paper, we particularly discuss results from one representative topology; where 49 mobile nodes were spread initially over a $7 \times 7$ unit grid on a scenario terrain of 1000 by 1000 meters [25] as shown in Figure 4. This is only initial position of nodes and it will move randomly based on the mobility model. The four corner nodes and the mid-nodes on each side of the square grid were chosen as sources and destination nodes as illustrated in Figure 5. Each source-destination pair had 2 CBR simultaneous active sessions in the opposite direction, thus giving rise to $16 \mathrm{CBR}$ flows as total of different sessions. The CBR is set to 10 packets per second and with 512 bytes of packet size. It came $15 \mathrm{sec}$ after the simulation started to give adequate time for exchanging routing messages [7]. The used radio type in our simulations is $802.11 \mathrm{~b}$ radio with Omni directional antenna model and $11 \mathrm{Mbps}$ data rate. To avoid the termination of a simulation due to the battery power exhaustion at the source or destination nodes, all source-destination pairs were configured to have maximum 


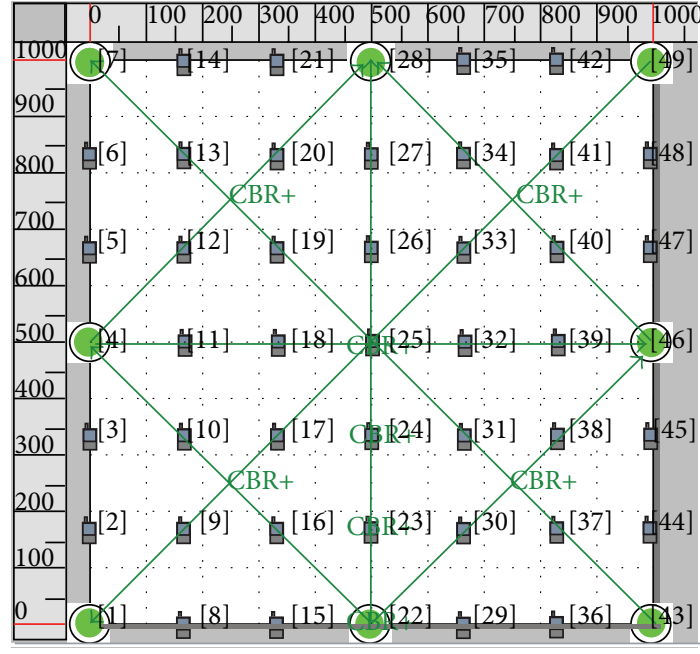

FIgURE 5: The simulation topology with 49 nodes and 16 CBR connections.

power resources $18[\mathrm{~mA} \cdot \mathrm{h}]$. The other "intermediate" nodes were configured with identical initial battery power levels 10 $[\mathrm{mA} \cdot \mathrm{h}]$. To study the performance of the proposed protocol, we performed experiments in two main simulation scenarios with simulation time of $400 \mathrm{sec}$. In the first scenario, the pause time of the nodes was changed from $50 \mathrm{sec}$ to $350 \mathrm{sec}$. However, in the second scenario, the pause time was set to $100 \mathrm{sec}$ and the maximum speed of the nodes was changed from $1 \mathrm{~m} / \mathrm{s}$ to $10 \mathrm{~m} / \mathrm{s}$. The wireless channel frequency was set to $2.4 \mathrm{GHz}$ and the radio transmission range was about $270 \mathrm{~m}$ as a result of the selected Wi-Fi parameters setting. All simulation scenarios were repeated 5 times with different randomly selected seeds to get different experiments, and the results were averaged over all experiments. The simulation parameters are presented in Table 1.

5.9. Performance Evaluation Metrics. The main aim of this work was to evaluate the energy efficiency and QoS metric of our enhanced MBA-OLSR routing protocol. For this purpose, the following metrics were considered.

Packet Delivery Ratio (PDR). The ratio of the data packets successfully received at the destination.

Average End-To-End Delay. It refers to the average of time duration over all surviving data packets that are transmitted from the source to the destination.

Network Life Time. The network lifetime is defined as the duration of time until the first node in a network fails due to the battery exhausted.

The Number of Dead Nodes. The metric gives the total number of dead nodes at the end of simulation time.

Energy Cost per Packet. This metric gives the ratio between the total consumed energy over the number of successfully received packets at the destinations.

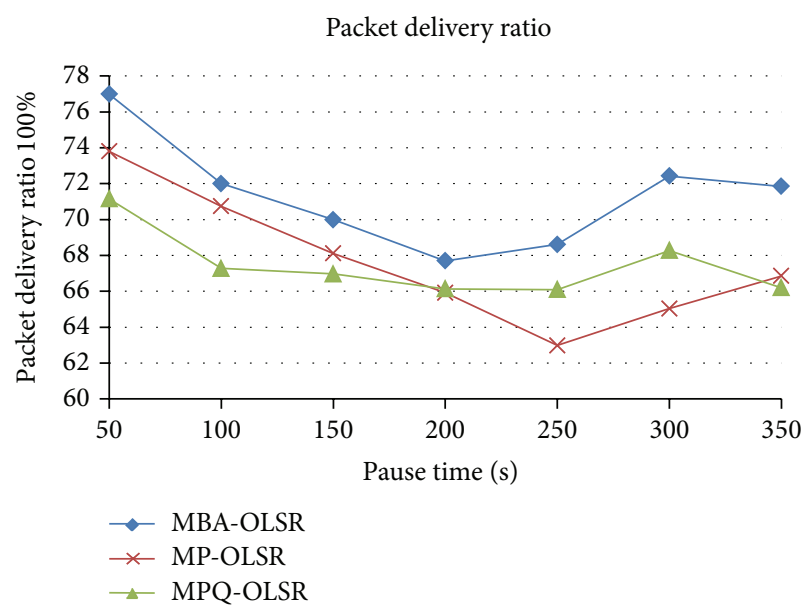

FIGURE 6: Packet delivery ratio for varying pause time.

TABLE 2: Number of dead nodes for varying pause time.

\begin{tabular}{lccccccc}
\hline \multirow{2}{*}{ Protocol } & \multicolumn{7}{c}{ Pause time [sec] } \\
& 50 & 100 & 150 & 200 & 250 & 300 & 350 \\
\hline MBA-OLSR & 2 & 8 & 10 & 15 & 10 & 16 & 12 \\
MP-OLSR & 3 & 9 & 8 & 15 & 13 & 15 & 13 \\
MPQ-OLSR & 4 & 9 & 14 & 19 & 19 & 16 & 16 \\
\hline
\end{tabular}

\section{MBA-OLSR Performance Evaluation}

The performance evaluation of the proposed protocol is described in this section. The results are analyzed and compared with MP-OLSR and MPQ-OLSR based on the considered metrics for energy efficiency and QoS. Our simulation scenarios were studied based on the mobility of the nodes. We considered two main simulation scenarios based on two parameters in the RWP mobility model, namely, pause time and maximum speed of nodes. We performed an extensive simulation and parametric analysis to show the behavior of the considered protocols in different scenarios.

6.1. Node's Pause Time Scenario. In order to evaluate the impact of node's pause time to the MBA-OLSR performance, this scenario presents a set of experiments, comparing MBAOLSR, MP-OLSR, and MPQ-OLSR, with the parameters listed in Table 1. Simulations were carried out by the varying nodes pause time from $50 \mathrm{sec}$ to $350 \mathrm{sec}$ increasingly by $50 \mathrm{sec}$ in each subscenario.

Figure 6 illustrates the packet delivery ratio of the three protocols. MBA-OLSR obtains higher delivery ratios up to $5 \%$ than both MP-OLSR and MPQ-OLSR regardless of pause time duration. Both MP-OLSR and MPQ-OLSR utilized nodes that provide the shortest path or lower congestion to destination without considering their battery resources and incur a higher number of dead nodes (as shown in Table 2), and therefore a lower data delivery ratio, particularly in longer pause time scenarios.

Figure 7 depicts the average end-to-end delay. Both MP-OLSR and MPQ-OLSR have higher end-to-end delay 


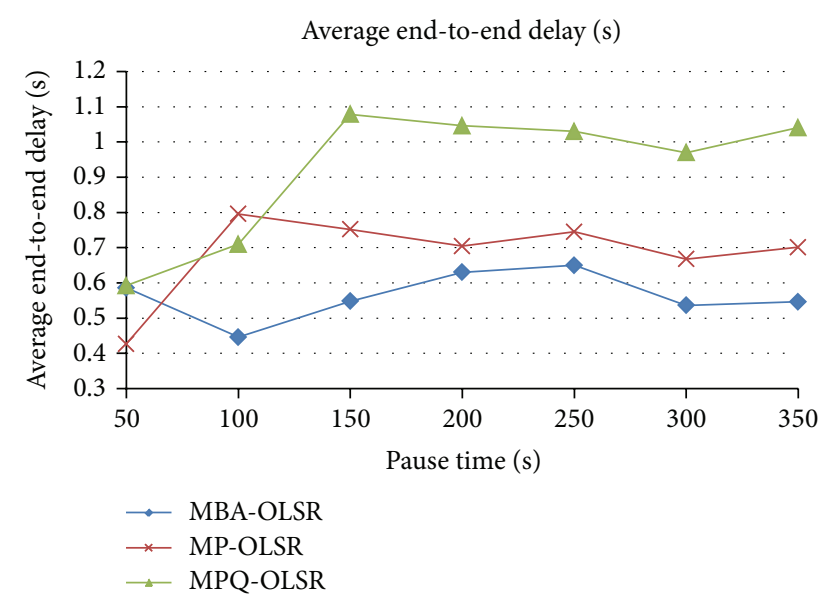

FIGURE 7: Average end-to-end delay for varying pause time.

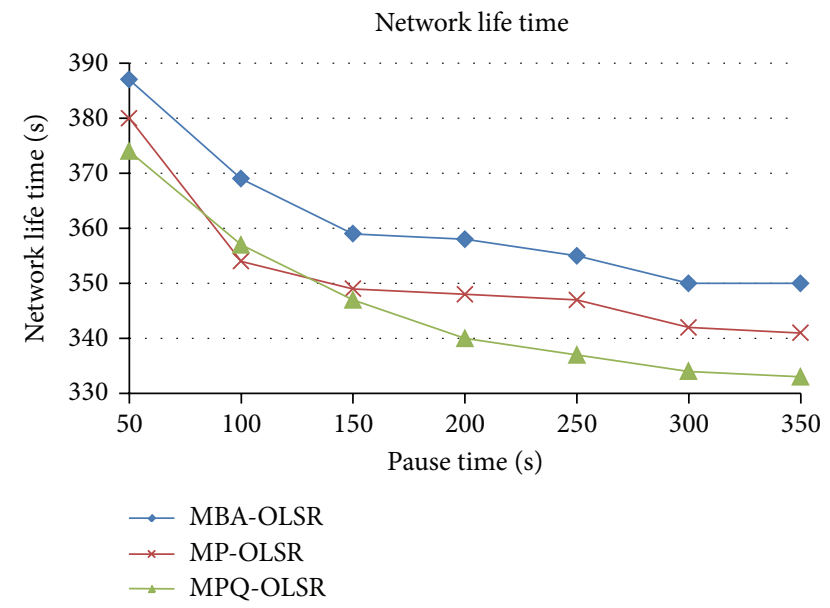

FIGURE 8: Network lifetime for varying pause time.

comparing with MBA-OLSR mostly because the dead intermediate nodes increase the delay to reach to the destination; unlike MBA-OLSR which exhibit shorter delays except in the first scenario.

In order to evaluate the energy efficiency metrics, Figures 8 and 9 show the network life time and energy cost per packet, respectively. The network life time with MBA-OLSR is decreasing as the duration of pause time increased. However, it performs better than both MP-OLSR and MPQ-OLSR in overall scenarios. This is because the MBA-OLSR tends to avoid intermediate nodes with low residual battery energy in its link cost function to find multiple paths to the destinations. Thus, the energy cost per successfully received packets also decreased as illustrated in Figure 9.

6.2. Node's Speed Scenario. This scenario evaluates the performance metrics of MBA-OLSR, MP-OLSR, and MPQ-OLSR in terms of speed of mobile nodes. The maximum speed in the RWP model changed from $1 \mathrm{~m} / \mathrm{s}$ to $10 \mathrm{~m} / \mathrm{s}$ and the pause time duration is set to $100 \mathrm{sec}$. Other simulation parameters are kept as same as Scenario 1. Figures 10 and 11 depict the

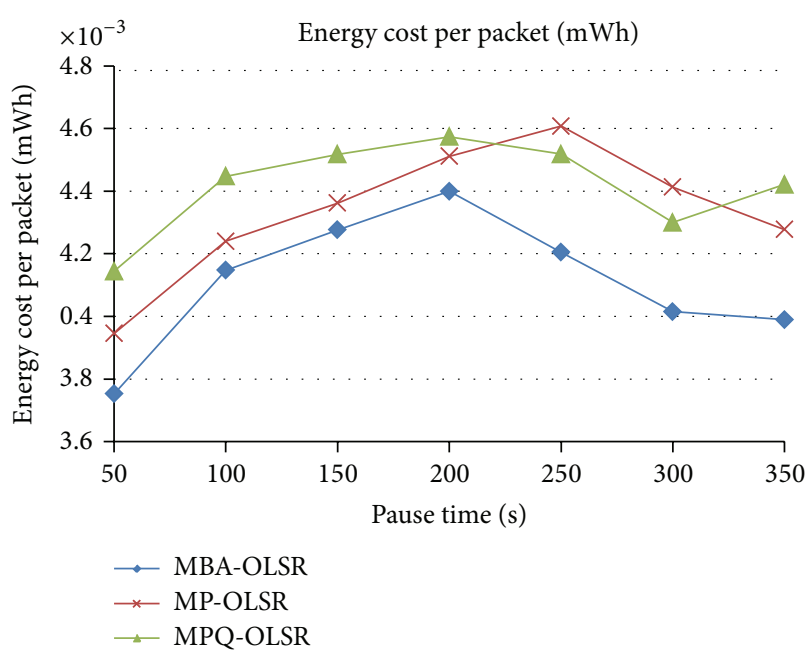

FIGURE 9: Energy cost per packet for varying pause time.

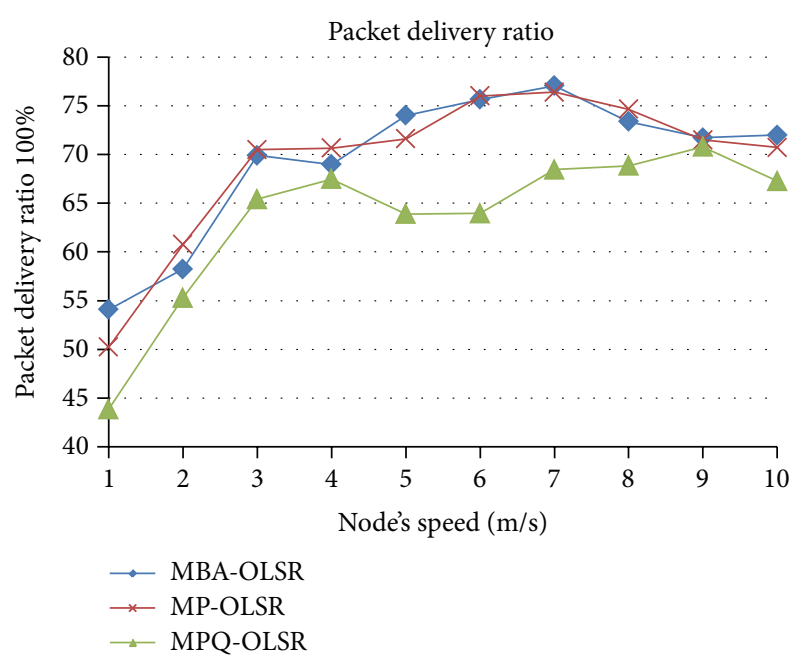

FIGURE 10: Packet delivery ratio for varying speed.

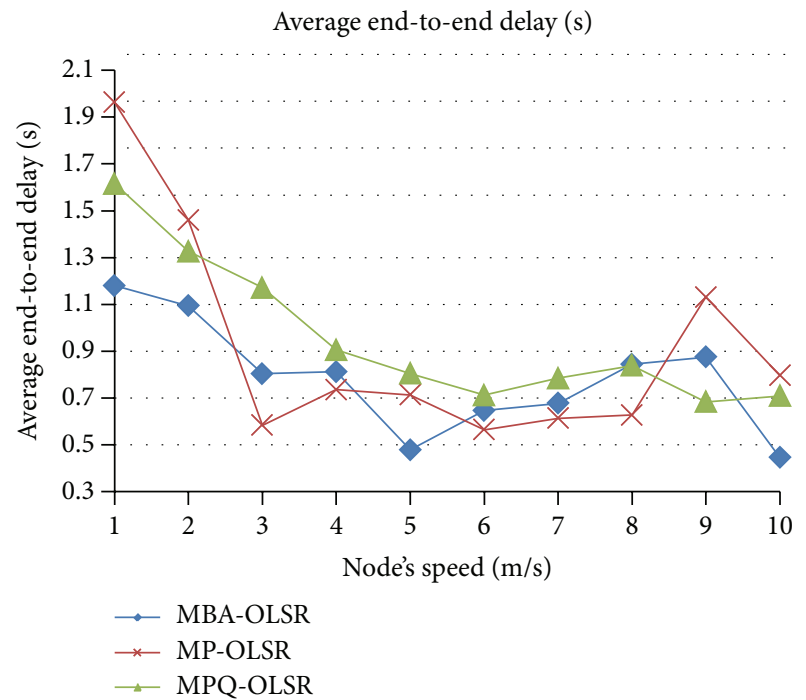

FIGURE 11: Average end-to-end delay for varying speed. 
TABLE 3: Number of dead nodes for varying speed.

\begin{tabular}{|c|c|c|c|c|c|c|c|c|c|c|}
\hline \multirow{2}{*}{ Protocol } & \multicolumn{10}{|c|}{ Node's speed $[\mathrm{m} / \mathrm{s}]$} \\
\hline & 1 & 2 & 3 & 4 & 5 & 6 & 7 & 8 & 9 & 10 \\
\hline MBA-OLSR & 22 & 28 & 24 & 18 & 18 & 15 & 16 & 7 & 10 & 8 \\
\hline MP-OLSR & 22 & 29 & 27 & 16 & 19 & 18 & 18 & 12 & 11 & 9 \\
\hline MPQ-OLSR & 22 & 29 & 26 & 18 & 21 & 18 & 16 & 6 & 11 & 9 \\
\hline
\end{tabular}

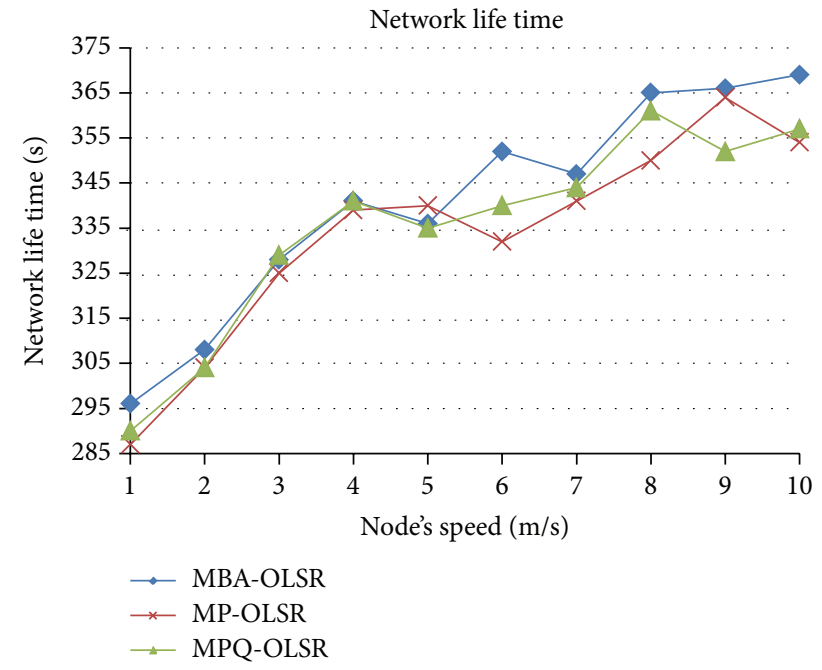

FIGURE 12: Network lifetime for varying speed.

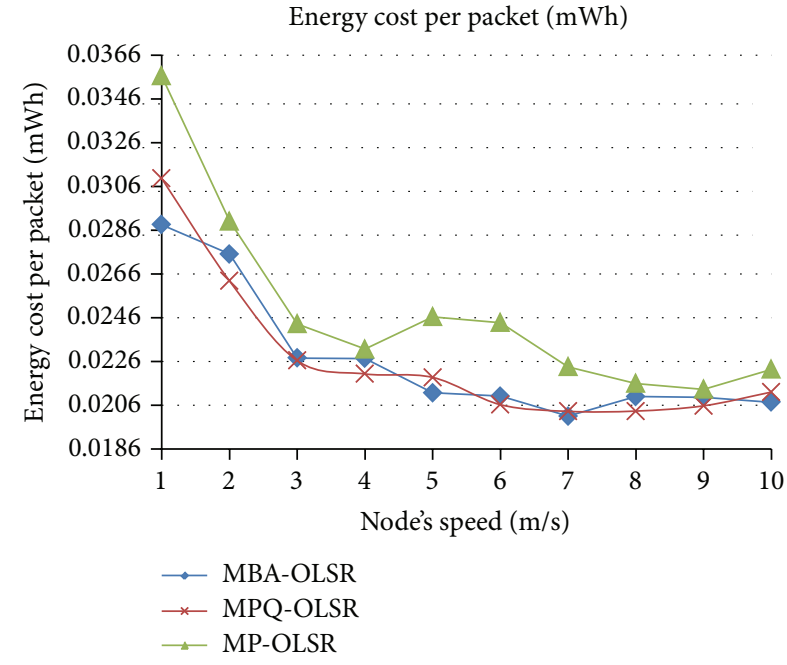

FIGURE 13: Energy cost per packet for varying speed. performance of the three protocols in terms of packet delivery ratio and average end-to-end delay. Both the MBA-OLSR and MP-OLSR obtain a higher packet delivery ratio than MPQ-OLSR mainly in the scenarios with the medium speed. In certain scenarios, the MBA-OLSR presents slightly lower packet delivery ratio as compared with the MP-OLSR because the node's mobility affects the exchanging of HELLO and TC messages between nodes to update the information about their residual battery energy. For the same reason, the MBAOLSR shows rather higher delay than MP-OLSR as shown in Figure 11. However, the number of nodes with exhausted battery in the MBA-OLSR is mostly less than both MP-OLSR and MPQ-OLSR as listed in Table 3.

The mobility impact on the network's life time and energy cost per packet is depicted in Figures 12 and 13, respectively. Obviously, the network lifetime is consistent for the three protocols, and it increased as the node's speed increased with slightly advances for the MBA-OLSR in overall scenarios. On the other hand, MBA-OLSR and MP-OLSR consumed less energy per packet than the MPQ-OLSR regardless of the node's speed as illustrated in Figure 13.

\section{Conclusions}

This study evaluated the performance of our protocol MBAOLSR which was compared with the MP-OLSR and MPQOLSR based on particular performance evaluation metrics. The proposed MBA-OLSR demonstrated a better performance than both the MP-OLSR and MPQ-OLSR in the pause time scenarios in terms of network lifetime and QoS metrics. It extended the network lifetime with achieving a lower end-to-end delay and a higher packet delivery ratio. However, in some speed scenarios, the MBA-OLSR performance degraded because of difficulties to update nodes with the level of battery energy of their neighbors. Moreover, the MPR selecting mechanism needs to be optimized because MPR nodes consume higher energy than others. Therefore, further enhancement of optimal energy based on MPR selecting mechanism will be developed with a compound node rank metric for better energy conservation and links quality.

\section{Conflict of Interests}

The authors declare that there is no conflict of interests regarding the publication of this paper.

\section{Acknowledgments}

The research was supported by Ministry of Higher Education (MOHE), Malaysia, under the Grant scheme no. LRGS/TD/2011/UKM/ICT/02/02.

\section{References}

[1] I. Chlamtac, M. Conti, and J. J.-N. Liu, "Mobile ad hoc networking: imperatives and challenges," Ad Hoc Networks, vol. 1, no. 1, pp. 13-64, 2003. 
[2] M. Bansal, R. Rajput, and G. Gupta, "Mobile ad hoc networking (MANET): routing protocol performance issues and evaluation considerations," 1999.

[3] T. Clausen, J. Yi, and A. C. de Verdiere, "LOADng: towards AODV version 2," in Proceedings of the 76th IEEE Vehicular Technology Conference (VTC Fall '12), pp. 1-5, Quebec City, Canada, September 2012.

[4] T. Clausen and P. Jacquet, "Optimized link state routing protocol (OLSR) RFC3626-OLSR,” 2003.

[5] T. Clausen, C. Dearlove, and P. Jacquet, "The optimized link state routing protocol version 2," draft-ietf-manet-olsrv2-00, Work in progress, 2006.

[6] M. A. hoc Networking and U. Herberg, "Integrity Check Value and Timestamp TLV Definitions for Mobile Ad Hoc Networks (MANETs) draft-ietf-manet-rfc6622-bis-03," 2013.

[7] J. Yi, A. Adnane, S. David, and B. Parrein, "Multipath optimized link state routing for mobile ad hoc networks," Ad Hoc Networks, vol. 9, no. 1, pp. 28-47, 2011.

[8] W. A. Jabbar, M. Ismail, and R. Nordin, "MBA-OLSR: a multipath battery aware routing protocol for MANETs," in Proceedings of the 5th International Conference on Intelligent Systems, Modelling and Simulation, pp. 630-635, Langkawi, Malaysia, 2014.

[9] T. Clausen, C. Dearlove, and J. Dean, RFC 6130 Mobile Ad Hoc Network (MANET) Neighborhood Discovery Protocol (NHDP), IETF, 2011.

[10] T. Clausen, C. Dearlove, J. Dean, and C. Adjih, "Generalized mobile ad hoc network (MANET) packet/message format," Internet Engineering Task Force, RFC, vol. 5444, 2009.

[11] T. Clausen and C. Dearlove, "RFC5497: Representing MultiValue Time in Mobile Ad Hoc Networks (MANETs)," Standards Track, 2009, http://www.ietf.org/rfc/rfc5497.txt.

[12] T. Clausen, C. Dearlove, and B. Adamson, "RFC5148: Jitter Considerations in Mobile Ad Hoc Networks (MANETs)," Informational, 2008.

[13] T. Clausen, "Internet Engineering Task Force U. Herberg Internet-Draft Fujitsu Laboratories of America Intended status: Standards Track R. Cole Expires,' US Army CERDEC, November 2013.

[14] J. Yi, "Protocole de Routage À Chemins Multiples Pour Des Réseaux Ad Hoc," 2010.

[15] F. de Rango, M. Fotino, and S. Marano, "EE-OLSR: energy efficient OLSR routing protocol for mobile ad-hoc networks," in Proceedings of the IEEE Military Communications Conference (MILCOM'08), pp. 1-7, San Diego, Calif, USA, November 2008.

[16] S. Mahfoudh and P. Minet, "An energy efficient routing based on OLSR in wireless ad hoc and sensor networks," in 22nd International Conference on Advanced Information Networking and Applications-Workshops (AINA '08), pp. 1253-1259, Okinawa, Japan, March 2008.

[17] Z. Guo, S. Malakooti, S. Sheikh, C. Al-Najjar, M. Lehman, and B. Malakooti, "Energy aware proactive optimized link state routing in mobile ad-hoc networks," Applied Mathematical Modelling, vol. 35, no. 10, pp. 4715-4729, 2011.

[18] R. D. Joshi and P. P. Rege, "Implementation and analytical modelling of modified optimised link state routing protocol for network lifetime improvement," IET Communications, vol. 6, no. 10, pp. 1270-1277, 2012.

[19] “EXata 3.1 Simulator," http://www.scalable-networks.com/products.
[20] C. E. Perkins, "Mobile ad hoc networking terminology," 1997, http://tools.ietf.org/html/draft-ietf-manet-term-00.

[21] I. Kang and R. Poovendran, "On Lifetime Extension and Route Stabilization of Energy-Efficient Broadcast Routing over MANET,' in Proceedings of the 3rd International Network Conference (INC '02), 2002.

[22] P. Rong and M. Pedram, "An analytical model for predicting the remaining battery capacity of lithium-ion batteries," IEEE Transactions on Very Large Scale Integration (VLSI) Systems, vol. 14, no. 5, pp. 441-451, 2006.

[23] C. Bettstetter, G. Resta, and P. Santi, "The node distribution of the random waypoint mobility model for wireless ad hoc networks," IEEE Transactions on Mobile Computing, vol. 2, no. 3, pp. 257-269, 2003.

[24] W. A. Jabbar, M. Ismail, and R. Nordin, "On the performance of The current MANET routing protocols for VoIP, HTTP," Journal of Computer Networks and Communications, vol. 2014, Article ID 154983, 16 pages, 2014.

[25] A. Misra and S. Banerjee, "MRPC: maximizing network lifetime for reliable routing in wireless environments," in Proceedings of the IEEE Wireless Communications and Networking Conference (WCNC'02), vol. 2, pp. 800-806, March 2002. 

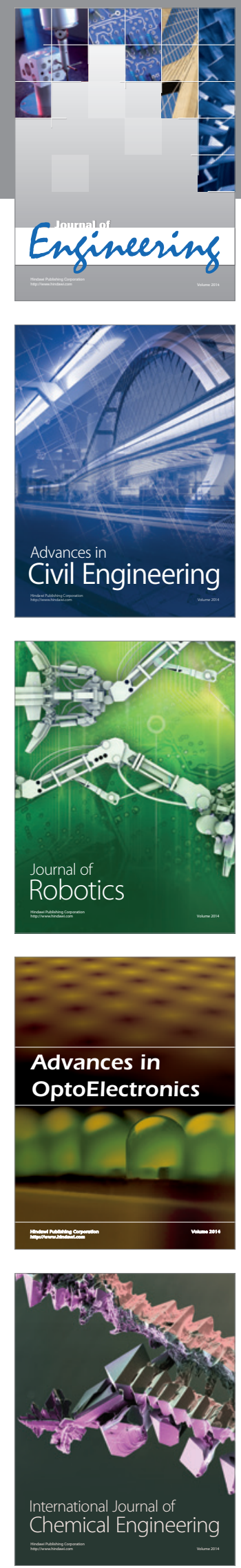

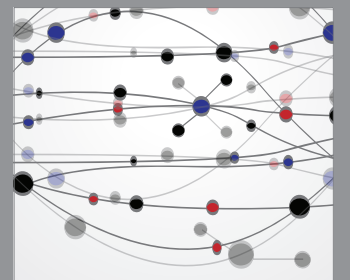

The Scientific World Journal
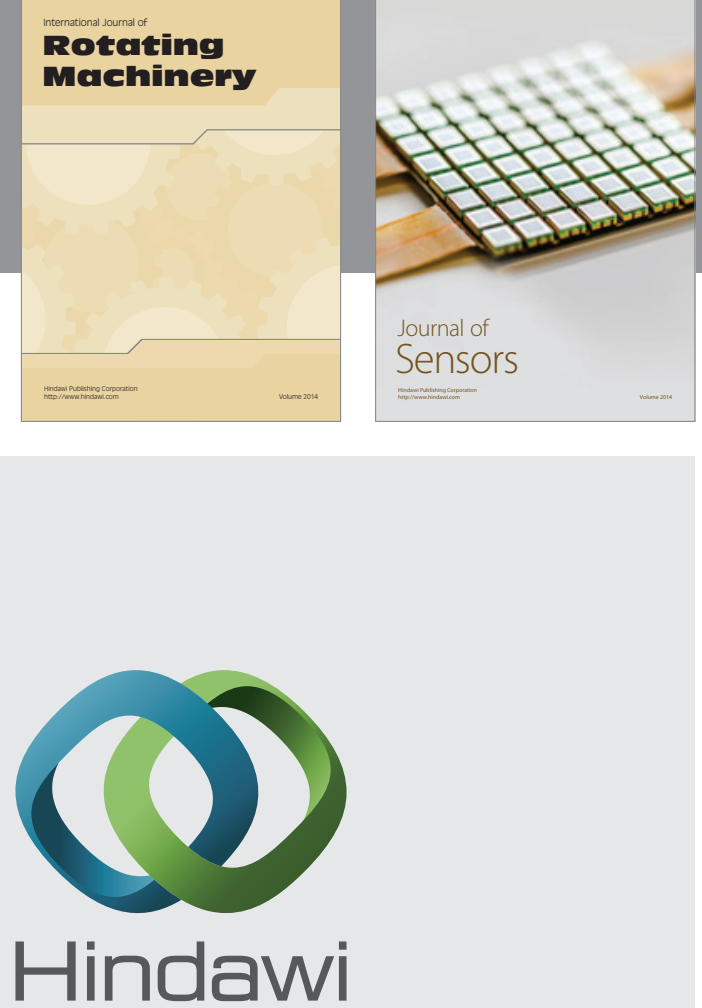

Submit your manuscripts at http://www.hindawi.com
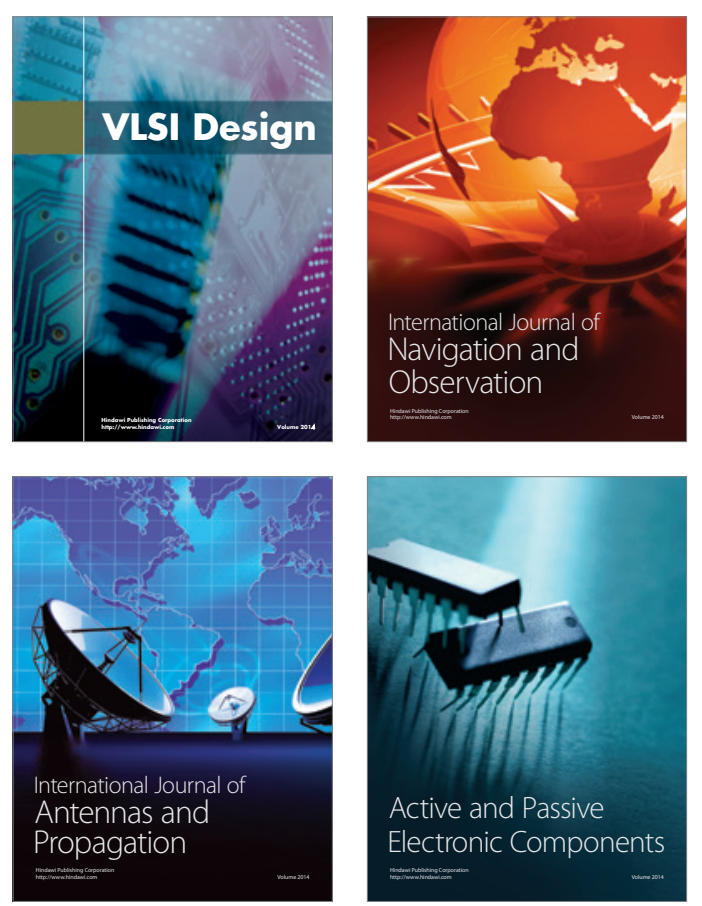
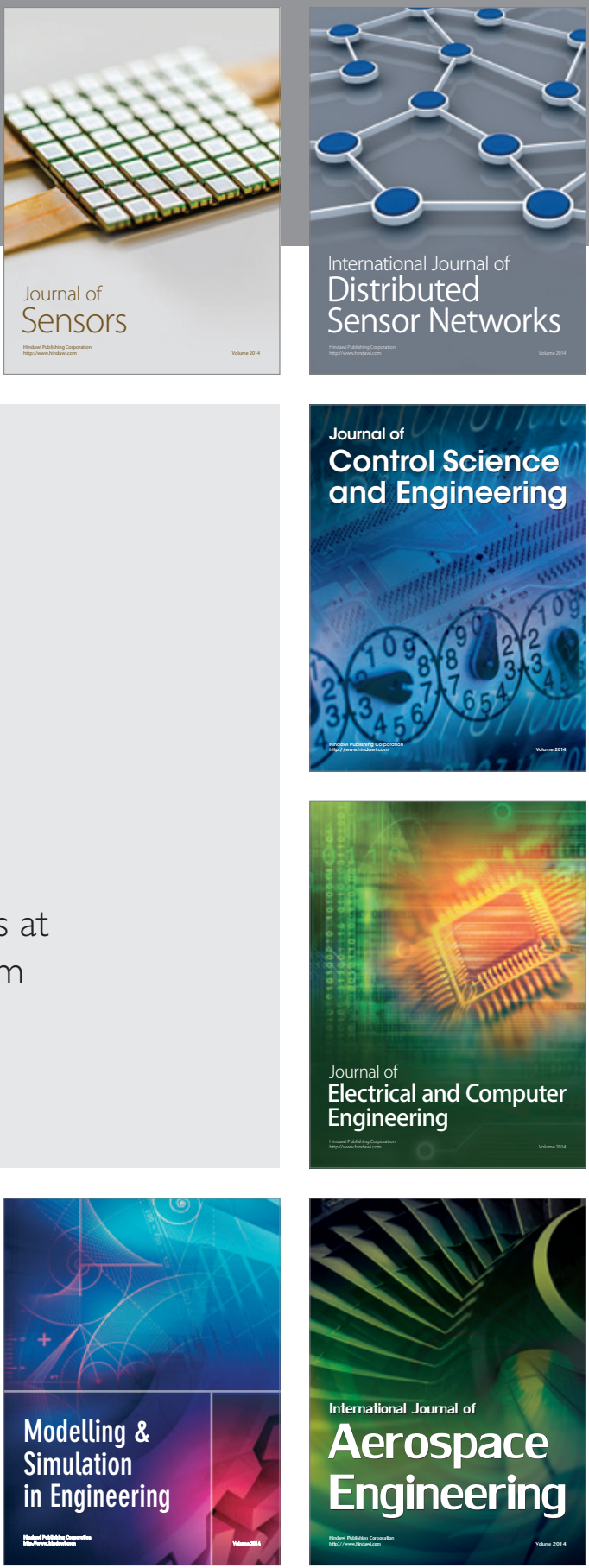

Journal of

Control Science

and Engineering
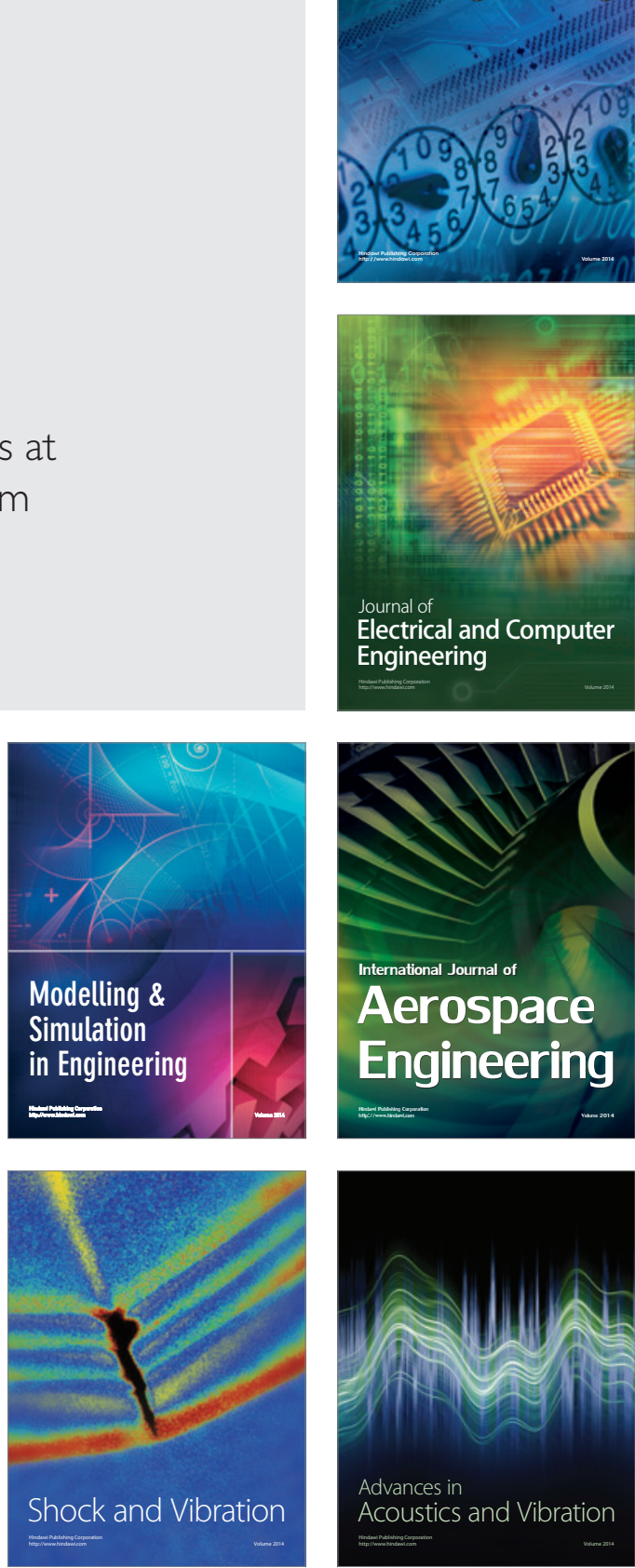